\title{
Changes in Cardiac Isomyosin Composition in an Iron Loading Anemia Rat Model
}

\author{
Wei Ting Cheng, Na Yoon Kim, Prashant Bhattarai, Murui Han, Archita Venugopal Menon, Jonghan Kim, Ban \\ An Khaw* \\ Department of Pharmaceutical Sciences, School of Pharmacy, Bouve College of Health Sciences, Northeastern University, Boston, MA. USA
}

Article Info

\section{Article Notes}

Received: May 28, 2019

Accepted: September 10, 2019

\section{*Correspondence:}

*Dr. Ban An Khaw, Ph.D., D.Sc. (Hon), Department of Pharmaceutical Sciences, School of Pharmacy, Bouve College of Health Sciences, Northeastern University, Boston, MA. USA; Email: b.khaw@northeastern.edu.

(c) 2019 Khaw BA. This article is distributed under the terms of the Creative Commons Attribution 4.0 International License.
Abstract

A rat model of iron loading anemia resulting in hypertrophic cardiomyopathy was used to assess the composition of cardiac isomyosin by ELISAs and immunohistochemistry relative to the chronological age of these animals and their normal controls. Cardiac myosin extracts of homozygous Belgrade $(b / b)$ rats that develop hypertrophic cardiomyopathy were compared to those from the heterozygous $(b /+)$ control rats at 4.5 and 11.5 weeks of age. Confirmation of the ELISA data in the increase in $\beta$-isomyosin in 11.5 weeks old $b / b$ rat hearts were obtained by immunohistochemical staining relative to 4.5 weeks old $b / b$ hearts and control 4.5 and 11.5 weeks old $b /+$ hearts. Quantitation and immunohistochemical demonstration of an increase in the $\beta$-isomyosin isoform can be confirmed in hypertrophic cardiomyopathy in iron loading anemia model.

\section{Introduction}

A cardiac hypertrophic homozygous Belgrade $(b / b)$ rat model with a thalassemic-like disorder of hypochromic microcytic anemia with hyperferritinemia and systemic iron loading results in the pathogenesis of cardiomyopathy. The autosomal recessive mutation in the Divalent Metal Transporter-1 (DMT-1) leads to impaired intestinal iron absorption and subsequent hemoglobin production for erythrocyte development $t^{1-3}$. DMT-1 regulates the transport of non-transferrin-bound iron (NTBI) $\mathrm{Fe}^{2+}$, manganese $\left(\mathrm{Mn}^{2+}\right)$ and cobalt $\left(\mathrm{Co}^{2+}\right)$ across the plasma membrane and out of the endosomal compartment $t^{4}$. A point mutation (G185R) in the SLC11A2 gene for DMT-1 in Belgrade rats results in anemia ${ }^{5}$. Homozygous Belgrade rats $(b / b)$ develop DMT-1 deficiency anemia, while heterozygous Belgrade $(b /+)$ rats are not susceptible to spontaneous iron deficiency anemia.

Deficiency in DMT-1 gives rise to anemia as well as promotes extensive liver iron loading in $b / b$ rats on a diet containing iron, whereas $b /+$ rats are not affected ${ }^{1,2}$. Hypochromic microcytic anemia increases cardiac output and has been correlated with the development of hypertrophic cardiomyopathy ${ }^{6}$. Cardiac hypertrophy in $b / b$ rats is associated with aging. This animal model of cardiac hypertrophy is correlated to changes in cardiac isoforms of myosin heavy chains (MHC). Such changes in $\mathrm{MHC}$ isoform concentrations with increasing age have been reported as percentage change of $\beta$-MHC in the Belgrade animal model ${ }^{7}$. In general isomyosin concentrations were determined by utilizing gel electrophoresis ${ }^{8}$, that is only qualitative, at best semi quantitative. 
Recently, Sukumaran and co-workers reported changes in cardiac isomyosin forms in iron overloading in hyperferremia deficient (Hfe-deficient) mice, a model of human hemochromatosis 9 . The authors showed that cardiac hypertrophy in 12-month old Hfe-deficient mice is associated with decrease in the $\alpha$ isomyosin relative to $\beta$ isoform of cardiac myosin. This change was demonstrated to be related to age in this model of Hfe-deficiency hemochromatosis. We now use a different model of cardiomyopathy and quantitated changes in the isoforms of cardiac myosin in Belgrade $(b / b)$ rats with aging using ELISA specific for $\beta$-isomyosin and total cardiac myosin specific monoclonal antibodies. Immunohistochemical quantiation was also performed with standard horse radish peroxidase (HRP) conjugated secondary antibody as well as with the Monster HRP reagent (GAM-HRP+, Akrivis LLC, Cambridge, MA) to amplify the chromogenic signal to demonstrate an increase in the immunohistochemical staining signal.

\section{Materials and Methods}

\section{Monoclonal antimyosin antibodies}

Monoclonal antimyosin 2G42D7 binds to all myosin whereas R11D10 is specific for the $\beta$-iso-myosin ${ }^{10,11}$. Monoclonal antibodies in culture media were used directly or as protein $\mathrm{G}$ affinity purified antibodies ${ }^{10}$. The hybridomas were cultured as previously reported ${ }^{10}$.

\section{Demonstration of cardiac iron overload and cardiac hypertrophy in experimental Belgrade and Heteral rats}

Anemic homozygous Belgrade $(b / b)$ rats and healthy heterozygous Belgrade $(b /+)$ rats (6-7 weeks old) were fed iron-supplemented diet (500 mg iron/kg diet) for 5 weeks and euthanized to assess various parameters at 11.5 weeks of age. The total iron concentration and non-heme iron concentration were determined by total iron and non-heme iron assay respectively ${ }^{12-14}$. The concentration of heme iron was obtained by subtracting non-heme iron concentration from the total iron concentration.

\section{Cardiac tissue extracts}

Canine cardiac myosin (Dog heart myosin [DHM]) was prepared as previously described from Canine hearts ${ }^{15}$.

The $b / b$ and $b /+$ rat hearts stored in liquid nitrogen were thawed on ice, and each heart was cut into small pieces and then was homogenized using a hand-held homogenizer (POLYTRON® ${ }^{\circledR}$ PT 1200 E, Kinematica). The homogenate was subjected to protein extraction by addition of 3-4 times the volume of the tissue homogenate of Radioimmunoprecipitation assay (RIPA) buffer [50 mM Tris $\left(4^{\circ} \mathrm{C}\right), 0.1 \%$ SDS, $1 \% \mathrm{NP}_{40}$ (Tergitol®), $0.5 \%$ Sodium Deoxycholate, and Protease Inhibitor Cocktail $\left.\left(4^{\circ} \mathrm{C}\right)\right]$. The homogenate was centrifuged at $16000 \mathrm{xg}$ for 6 minutes at $4^{\circ} \mathrm{C}$. The supernatant was collected and stored at $-80^{\circ} \mathrm{C}$ for future use.

\section{Estimation of the protein concentration of rat heart supernatants of the homogenates by the Bradford Method}

Determination of the concentration of $b / b$ and $b /+$ rat heart supernatant solutions was by the Bradford Method ${ }^{16}$. Aliquots of $978 \mu \mathrm{l}$ of the Bradford Reagent were added to serial dilutions of BSA in PBS $(15,10,5,2.5$, and $0 \mu \mathrm{g} / \mathrm{ml})$ and were used to generate the standard curve. Aliquots $(2$ $\mu \mathrm{l}$ ) of $b / b$ or $b /+$ rat heart samples and $20 \mu \mathrm{l}$ of PBS were added to additional wells of the assay plate respectively with $978 \mu \mathrm{l}$ of Bradford Reagent. The plates were incubated for 5 minutes and the optical density (OD) read at 595 $\mathrm{nm}$, using a spectrophotometer (Epoch Biotek ${ }^{\circledR}$ ). The total protein concentration of $b / b$ and $b /+$ rat heart samples were extrapolated from the formula of the BSA standard curve.

\section{ELISAs}

Determination of the concentration of total $\mathrm{MHC}$ and $\beta$-MHC of $b / b$ and $b /+$ rat heart supernatant samples was performed by ELISAs using the two cardiac myosyinspecific monoclonal antibodies 2G42D7 and R11D10. Standard curve for the ELISAs utilized canine (dog) heart myosin (DHM) for quantitation ${ }^{17}$. Both myosyin-specific monoclonal antibodies were prepared as described previously ${ }^{10}$. DHM was previously prepared in our laboratory.

To ensure reproducibility and accuracy, 6 standard curves were performed under the same conditions with each antibody. The mean \pm 1 SD (error bars) are shown in Figure 1. Maximum binding of each standard curve was then normalized to represent $100 \%$. The formula of the normalized standard curves was obtained by fitting of 4-parameter logistics, using ReaderFit (MiraiBio Group of Hitachi Solutions America, Ltd).

Since DHM is primarily $\beta$-isomyosin, both normalized standard curves were almost identical with no significant differences (Figure 1).

Determination of the concentration of total MHC and $\beta$-MHC in $b / b$ and $b /+$ rat heart samples was by ELISAs ${ }^{18,19}$ using two monoclonal antimyosin antibodies ${ }^{15,20}$. Both myosin specific monoclonal antibodies were prepared as described previously ${ }^{15}$. 96-well microtiter plate $\left(\right.$ Fisherbrand ${ }^{\circledR}$ ) was coated with $3.1 \mathrm{mg} / \mathrm{ml}$ of Belgrade or $b /+$ rat heart extracts, however the OD reading was too low. To obtain accurate determination of the $\beta$-MHC concentrations of homogenized $b / b$ and $b /+$ rat heart extracts, the maximum absorbance was normalized as described for normalization of the binding of DHM. 


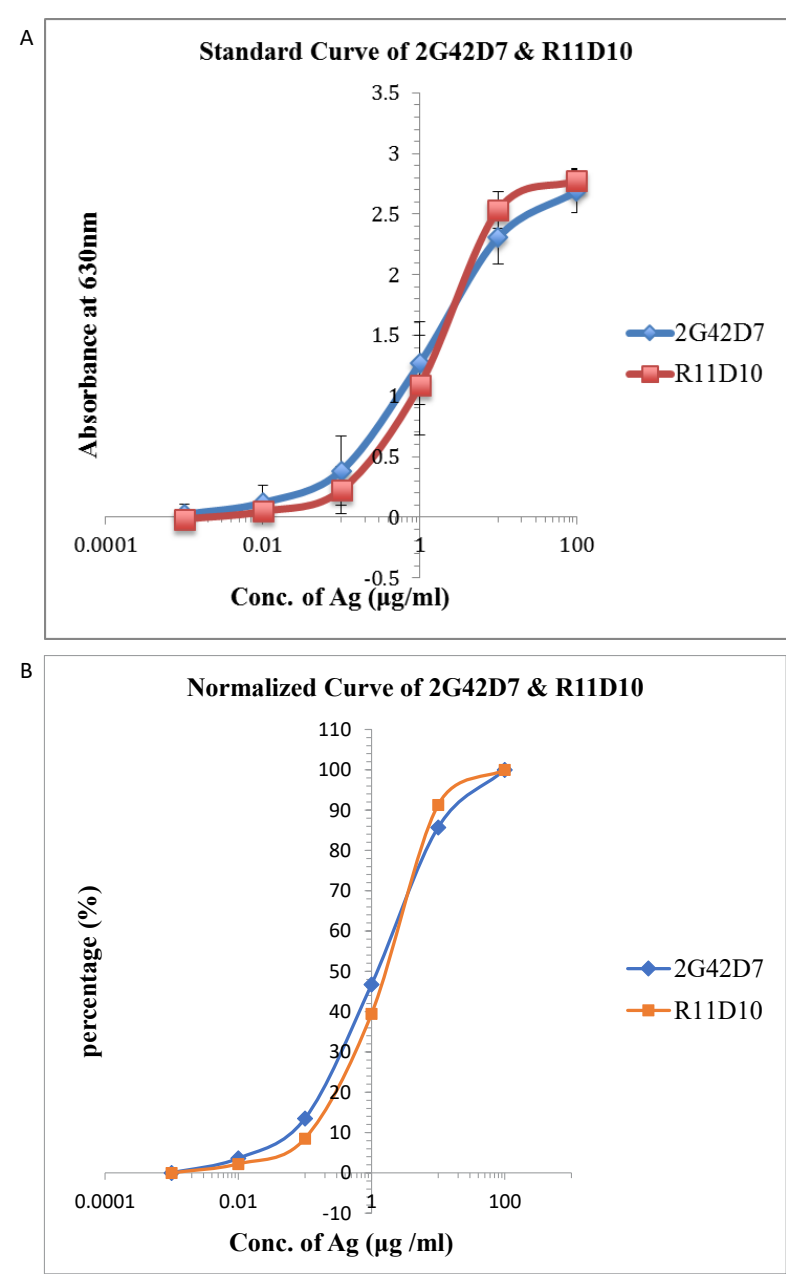

Figure 1. Standard curves of antimyosin R11D10 ( $\beta$-isomyosin specific) and 2G42D7 (total myosin) monoclonal antibodies by ELISA are shown as absorbance (top panel) as normalized curves (bottom panel).

\section{Immunohistochemical analysis of frozen rat heart sections}

\section{Preparation of frozen rat heart sections}

Distribution of total MHC and $\beta$-MHC of $b / b$ and $b /+$ rat heart samples by immunohistochemical staining was by using the same two myosin-specific monoclonal antibody 2G42D7 and R11D10 ${ }^{15,20}$.

Rat hearts were collected from exsanguinated rats and each heart was placed in a tissue holder that had been filled with the cryoembedding compound (Tissue-Tek ${ }^{\circledR}$ O.C.T. $)^{21}$. The tissue holders were stored at $-80^{\circ} \mathrm{C}$ until used. The frozen heart sections were prepared by using the Cryostat Microm HM550 (Thermo Scientific, Kalamazoo, MI.). The temperature of the cryochamber was set at $-20^{\circ} \mathrm{C}$. The specimen disk was built up with O.C.T. and a section of the frozen heart was mounted and returned to the cryochamber. Then the O.C.T. compound was added on top of the frozen heart section to cover the whole tissue.
The specimen disk was allowed to freeze for 5 minutes. The thickness of the heart sections was set at 10 microns. The mounting angle of the heart was set at position 6 . The vacuum pressure of the cryostat was set at 9.5. The cryosections were mounted on clean microscope slides and the sections were dried overnight at RT. The sections were stored in a sealed slide box at $-80^{\circ} \mathrm{C}$ until used. Hearts from 3 rats each of 4.5 and 11.5 weeks old $b / b$ and 2 and 3 hearts from 4.5 and 11.5 weeks old $b /+$ rats were sectioned into multiple cryosections for immunohistochemical staining.

Immunohistochemical assessment of rat heart sections by GAM-HRP (MP Biomedicals, LLC) and GAMHRP+ (AKRIVIS, Z-TECT ${ }^{\mathrm{TM}}$ )

Slides containing the frozen heart sections were immersed in pre-cooled acetone $\left(-20^{\circ} \mathrm{C}\right)$ for 10 minutes to fix the sections ${ }^{21}$. Then the slides were removed and the acetone was allowed to evaporate from the tissue sections for 20-30 minutes at RT. Each slide was washed 3 times with $0.1 \mathrm{M}$ PBS, 5 minutes each. Then the tissue sections were incubated with $100 \mu \mathrm{l}$ of $3 \%$ BSA in a moisture chamber for 1 hour at room temperature to block nonspecific binding. The slides were washed 3 times with $0.1 \mathrm{M}$ PBS, 5 minutes each. Then aliquots of $100 \mu \mathrm{l}$ of 10 $\mu \mathrm{g} / \mathrm{ml}$ of the primary antibody 2G42D7 or R11D10 were added to the tissue sections on the slides respectively and the slides were incubated in moisture chambers at $4^{\circ} \mathrm{C}$ for 24 hours. Next day, the slides were washed three times with $0.1 \mathrm{M}$ PBS-T, followed by three times with $0.1 \mathrm{M}$ PBS, for 5 minutes each wash. After washing, aliquots of 100 $\mu \mathrm{l}$ of 1:1000 dilution of GAM-HRP (MP Biomedicals, LLC) or 1:40 dilution of GAM-HRP+(AKRIVIS, Z-TECT ${ }^{\mathrm{TM}}$ ) were added respectively to the sections on the slides and the slides were incubated in the moisture chamber for four hours at RT. The dilutions used were normalized to contain the same concentration of the secondary goat anti-murine antibody. The slides were then washed three times with 0.1 M PBS-T and followed by three times with $0.1 \mathrm{M}$ PBS. Each incubation was for 5 minutes. Then aliquots of $100 \mu \mathrm{l}$ of diamino benzedine (DAB) substrate solution (freshly made just before use: $0.05 \%$ DAB with $0.015 \% \mathrm{H}_{2} \mathrm{O}_{2}$ in $0.1 \mathrm{M} \mathrm{PBS}$ ) were applied to the sections on the slides to develop the color of the chromogen. The color was allowed to develop for about $50 \mathrm{~min}$. The slides were then washed three times with $0.1 \mathrm{M}$ PBS for 5 minutes each. The slides were then dehydrated with serial alcohol treatment in 95\% (two times) and 100\% (two times) alcohol solutions. About 100 $\mu$ l of Permount ${ }^{\circledR}$ (Fisher Scientific) was added to each tissue section. Cover slip was applied and sealed with clear nail polish. After the nail polish had dried, the mounted slides were stored in $-20^{\circ} \mathrm{C}$ in a sealed slide box for subsequent microscopic examination. Bright-Field microscopy (Nikon Eclipse E400) was set to the following conditions: a) Ocular: CFI 10X/22M, b) Objective: Plan 40X/ 0.65 PH2 DL 
Table 1. Various parameters in 11.5 -week-old $b / b$ and $b /+$ rats.

\begin{tabular}{|l|l|l|l|l|}
\hline Parameter (Unit) & $\mathrm{b} /+$ & $\mathrm{b} / \mathrm{b}$ & $\mathrm{N}$ (each) & P value \\
\hline Body weight $(\mathrm{g})$ & $256.0 \pm 1.8$ & $219.5 \pm 8.4$ & $10-12$ & $<0.001$ \\
\hline Heart/body weight ratio $(\%)$ & $0.30 \pm 0.01$ & $0.48 \pm 0.03$ & 4 & $<0.001$ \\
\hline Total Fe in heart $(\mu \mathrm{g} / \mathrm{g})$ & $99.76 \pm 7.56$ & $78.45 \pm 11.24$ & 4 & 0.020 \\
\hline Non-heme Fe in heart $(\mu \mathrm{g} / \mathrm{g})$ & $36.80 \pm 8.29$ & $37.04 \pm 6.45$ & 5 & 0.960 \\
\hline Ratios of Non-heme/Total Fe & $36.39 \pm 9.68$ & $48.89 \pm 6.45$ & 3,4 & 0.246 \\
\hline
\end{tabular}

( $\infty / 0.17$, WD 0.57), c) Digital micrographs were acquired at an exposure time of $21.151 \mathrm{~ms}$ and the Gain was set at 2, d) White Balance was set at 3.783, 2.246, 1, e) Color Enhancement: Gamma 2.53; Contrast 0.8; Saturation 0.67; Color temperature 10273 , f) Acquired image size was 1600 X 1200 pixels, g) Bit Depth: 24 bpp (RGB), h) Sensor Clear Mode: Continuous, i) Chip Area: Full Chip. The intensity of the immunoperoxidase stained images were analyzed by Image (NIH Image).

\section{Statistics}

All data were analyzed using Student's $t$-test. The p-value $<0.05$ is set as a measure of statistical significance. ELISA data were analyzed using Gen 5.0 software for mean and standard deviation. Images of immunohistochemical studies were analyzed using ImageJ (NIH Image). Data are presented as means \pm 1SD and were analyzed using unpaired Student's $t$-test.

\section{Results}

Table 1 shows the physiological parameters of the 11.5 week-old Belgrade rat hearts. Total iron concentration and non-heme iron concentration determined by total iron and non-heme iron assay respectively ${ }^{11-13}$ demonstrated that the ratio of non-heme iron relative to total iron (\%) was not significantly different between $b / b$ and $b /+$ rats. In the 11.5 weeks old $b / b$ rats, the total iron concentration was decreased to $78.5 \mu \mathrm{g} / \mathrm{gm}$ myocardium from $99.8 \mu \mathrm{g} /$ gm in normal $b /+$ rats, indicating the anemic condition in $b / b$ rats. Cardiac hypertrophy was observed in 11.5 weeks old Belgrade rats relative to normal $b /+$ rats (heart/body weight ratio $0.48 \pm 0.01 \%$ and $0.30 \pm 0.03 \%$ respectively, $\mathrm{p}<0.001 ; \mathrm{n}=4$ ).

Even though, total iron in the heart decreased in $b / b$ rats $(P=0.02)$, the ratio of non-heme to total iron was not significantly different relative to the control of $b /+$ rats. However, the heart/body weight ratios increased from $0.30 \pm 0.01$ to $0.48 \pm 0.03(\mathrm{P}<0.001)$. This may be due to decreased heme iron in the heart of the $b / b$ rats relative to age-matched $b /+$ rats. These parameters are consistent with the pathogenesis of hypertrophic cardiomyopathy.

Figure 2 A shows the $\% \beta$-isomyosin concentrations relative to total myosin of 4.5 and 11.5 weeks old $b / b$ $(16 \pm 7 \%$ and $38 \pm 5 \%$ respectively) and $b /+(17 \pm 2 \%$ and $19.5 \pm 2.5 \%$ respectively) hearts assessed by ELISA. There is no difference between the $\beta$-isomyosin concentration

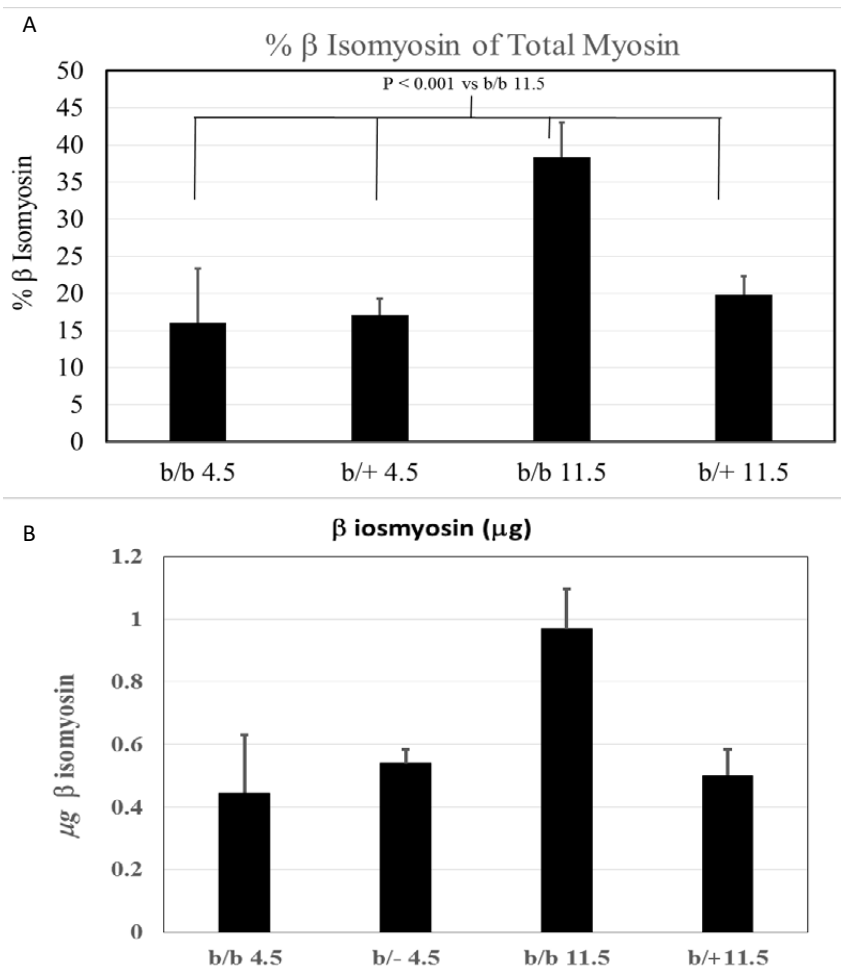

Figure 2. (A) shows the $\% \beta$-isomyosin in 4.5 and 11.5 weeks old homozygous Belgrade $(b / b)$ and healthy heterozygous $(b /+)$ rat hearts.

(B) The 11.5 weeks old $b / b$ hearts showed significantly higher concentration of $\beta$-isomyosin in $\mu \mathrm{g}$ concentration than in the comparator groups $(p<0.001)$.

relative to total myosin in 4.5 months old $b / b(16 \pm 7 \%)$ and $b /+(17 \pm 2 \%)$ rat hearts. There is also no significant difference between the 4.5 and 11.5 weeks old $b /+$ hearts $(17 \pm 2 \%$ and $19.5 \pm 2.5 \%$ respectively. Only the 11.5 months old $b / b$ hearts showed significantly higher percentage of $\beta$-isomyosin $(38.5+5 \%)$ than that of the comparators. Figure $2 \mathrm{~B}$ shows the normalized micrograms of $\beta$-isomyosin in 4.5 and 11.5 weeks old $b / b$ and $b /+$ rat hearts. The total myosin concentration was normalized to the highest value, then the $\beta$ isomyosin concentration was calculated from the normalized total myosin concentration. Only the concentration in the 11.5 weeks $b / b$ hearts is statistically significantly greater than the $\beta$-isomyosin concentrations in 4.5 weeks old $b / b$ and $b /+$ rats and the 11.5 weeks old $b /+$ hearts.

Figure 3 A shows that staining with 2G42D7 for total 

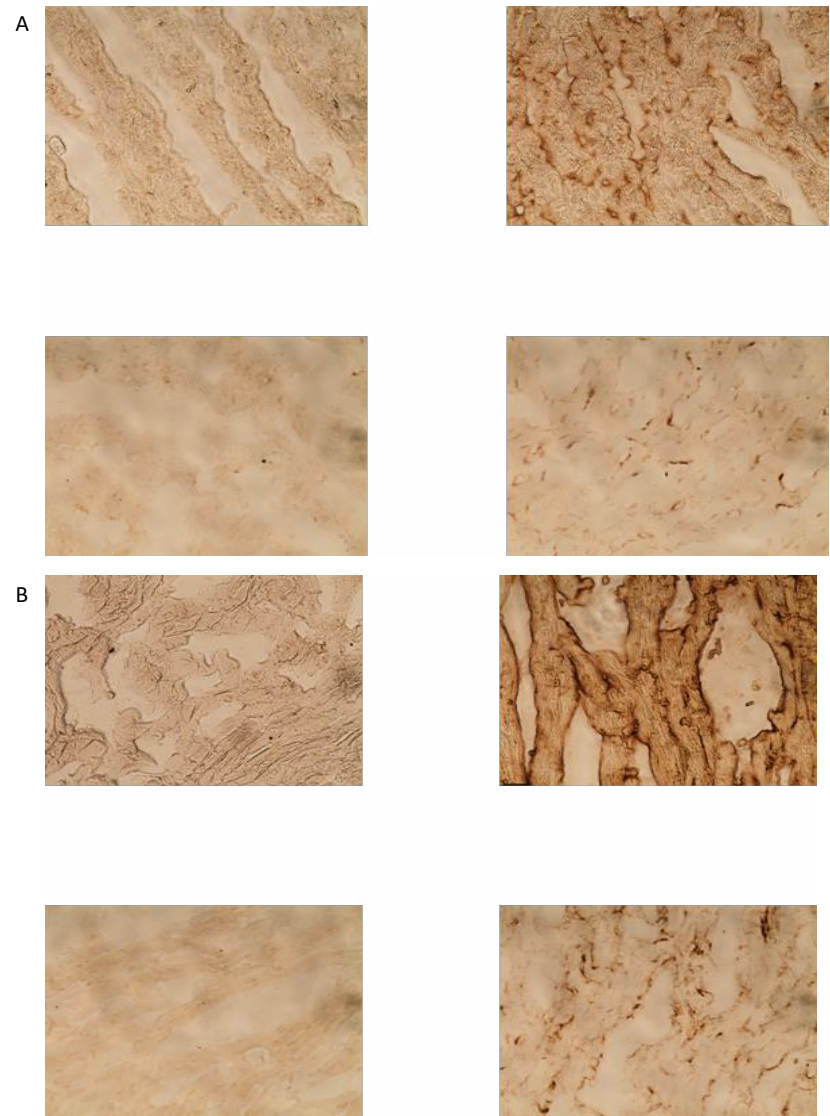

Figure 3. (A) shows the immunoperoxidase staining of 11.5 weeks old $\mathrm{b} /+$ heart frozen sections with non-specific murine IgG (top and bottom left panels), and with 2G42D7 (top right panel) and R11D10 (bottom right panel) using the secondary GAM-HRP antibody. (B) When GAM-HRP+ was substituted for GAM HRP as the secondary HRP conjugated antibody, the DAB signal was significantly increased for staining with both 2G42D7 and R11D10.

myosin and R11D10 for $\beta$-isomyosin followed by using the commercially available GAM-HRP is higher than that of the background activity obtained using murine IgG as controls. However, when GAM-HRP was substituted with GAM-HRP+ from Akrivis LLC, the intensity of staining with 2G42D7 as well as with R11D10 was increased (B). The IgG control background activity however, remained the same as those obtained with GAM-HRP (A left panes) or GAM-HRP+ (B left panels).

Figure 4 show the immunoperoxidase staining of $b / b$ (top row) and $b /+$ (bottom row) frozen heart sections with mouse IgG (top left panel), 2G42D7 (middle panels) and R11D10 (right panels) developed with GAM-HRP+. The intensity of the background non-specific activity was the same in the $b / b$ or $b /+$ sections reacted with murine IgG and 2G42D7, the latter showing the same intensity of staining of total myosin irrespective of whether the sections were from $b / b$ (top middle panel) or $b /+$ rat (bottom middle panel) hearts. Staining with R11D10 however, showed that there was more chromophore localization in the $b / b 11.5$
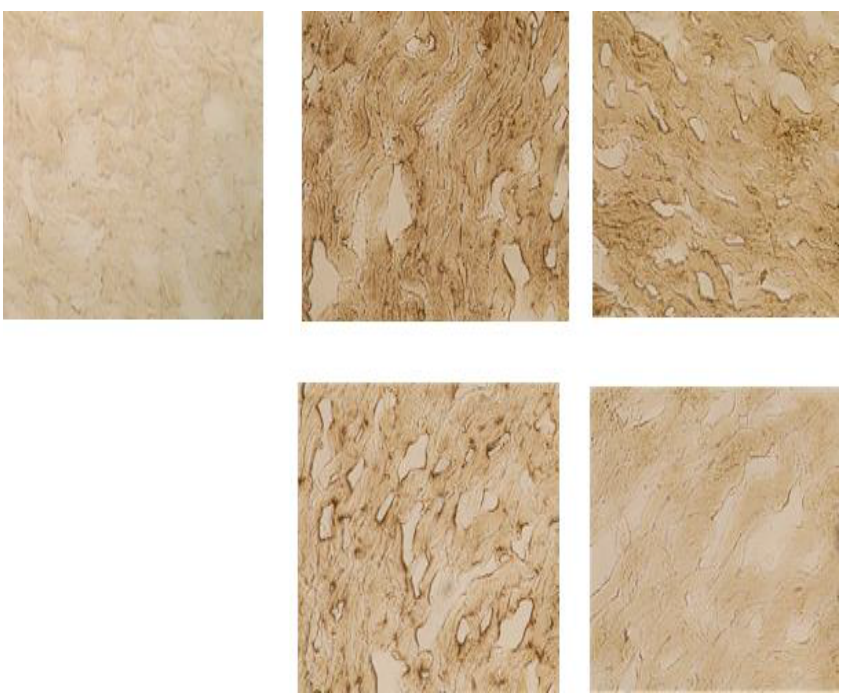

Figure 4. Immunoperoxidase stained Belgrade hearts with 2G42D7 (top middle panel) and R11D10 (top right panel) compared to $b /+$ heart sections stained with 2G42D7 (bottom middle panel) and R11D10 (bottom right panel). Control was stained with normal murine IgG (left panel).

weeks old heart section (top right panel) than in the b/+ heart section (bottom right panel) indicating that there was an increase in the $\beta$-isoform of cardiac myosin in the 11.5 weeks old $b / b$ rat hearts.

Figure 5 shows that non-specific background activity with IgG was the same with the secondary GAM-HRP or GAM-HRP+. Signal amplification was obtained when GAMHRP+ was used with 2G42D7 or R11D10 antibodies to stain the heart sections. Comparison in 4.5 and 11.5 weeks old $b / b$ heart sections stained with GAM-HRP to $b /+11.5$ weeks heart section showed that the intensity of staining of total myosin was the same (Figure 6, p=ns). However, the frozen heart sections stained with R11D10 showed different intensity of staining between 11.5 and 4.5 weeks old $b / b$ Belgrade heart sections ( $\mathrm{p}<0.01$ ). This difference is also seen between 11.5 weeks old $b / b$ heart sections and 4.5 and 11.5 weeks old $b /+$ heart sections $(\mathrm{p}<0.01)$ (Figure $7)$. Figure 8 shows the $\% \beta$-isomyosin of total cardiac myosin is the same in 4.5 and $11.5 \mathrm{~b} /+$ and 4.5 weeks old $b / b$ rat hearts but the $\beta$-isomyosin concentration is much higher in 11.5 weeks old $b / b$ hearts. This is consistent with much larger heart size along with increased ventricular wall thickness in 11 weeks old $b / b$ rats compared to $b /+$ rats (Figure 9).

Even though the absorbance in the 4.5 weeks old $b /+$ heart sections for total myosin shown in Figure 6 is a little lower than the absorbance for all other total myosin assessments, when the \% value was calculated for the $\beta$-isomyosin in the 4.5 months old $b /+$ and $b / b$ hearts and 11.5 weeks old $b /+$ hearts, the $\%$ is not significantly different. However, these values are significantly lower 


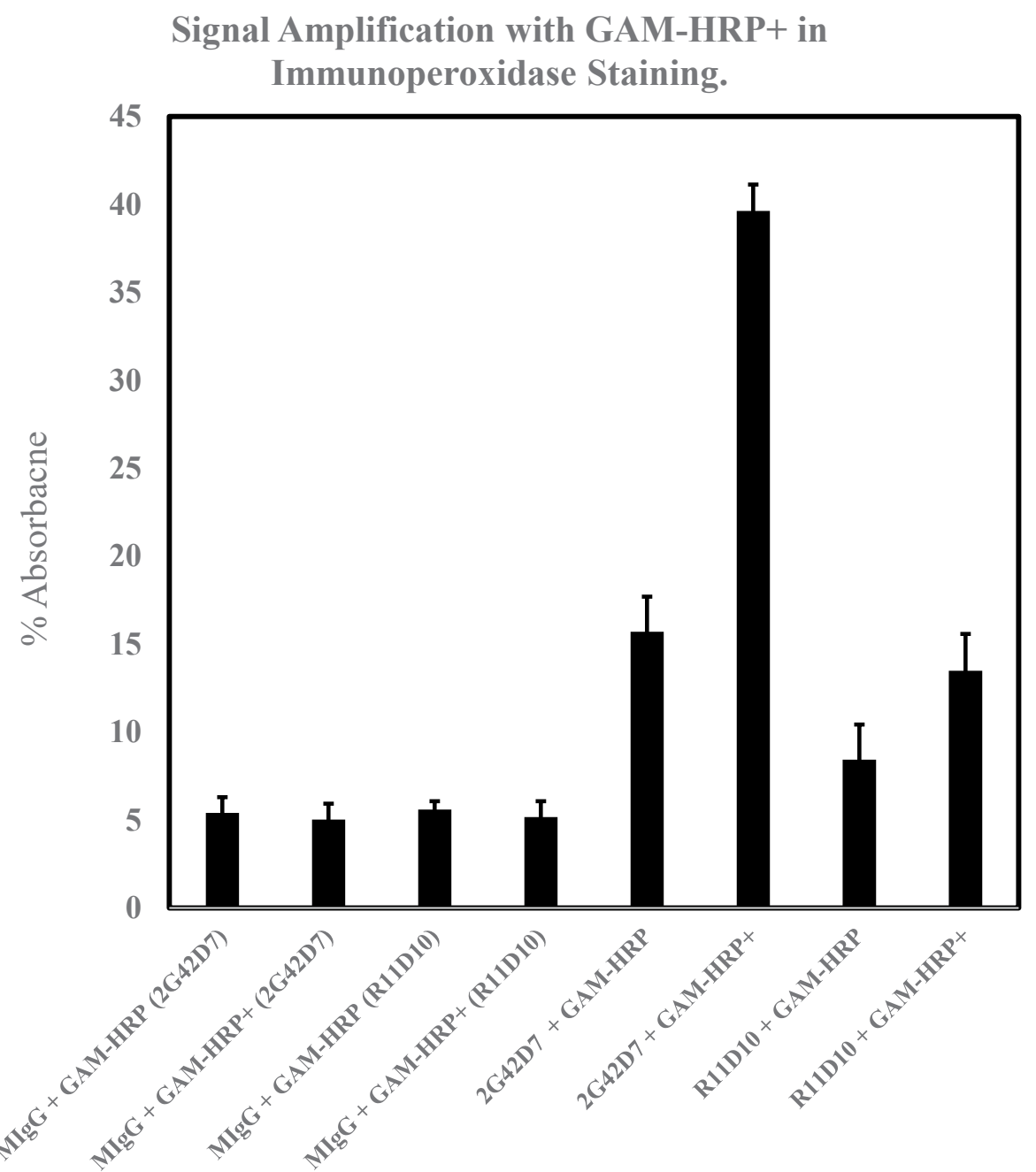

Figure 5. Shows the quantitation of absorbance from immunohistochemical staining of control $b /+$ rat hearts with Murine IgG to establish non-specific adsorption and with 2G42D7 and R11D10 monoclonal antibodies with secondary GAM-HRP or GAM-HRP+. As can be seen there is no difference in the non-specific absorbance whether GAM-HRP or GAM-HRP+ was used. However, when 2G42D7 or R11D10 monoclonal antimyosin antibodies were used, the signal generated in heart sections counter stained with GAM-HRP+ is significantly greater than that achieved with the standard GAM-HRP. However, subsequent immunohistology to quantitate $\beta$-isomyosin in $b /+$ and $b / b$ rat hearts were undertaken using only GAM-HRP as the secondary antibody which is commercially readily available.

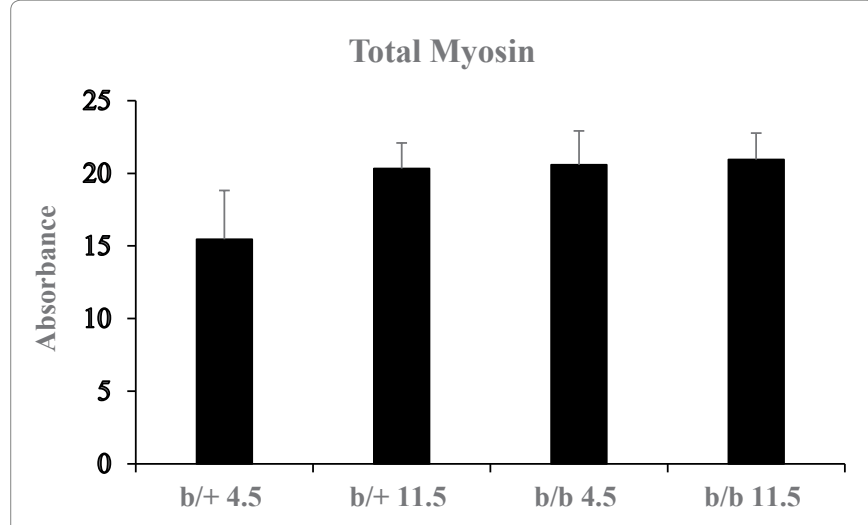

Figure 6. Absorbance of histochemical stained sections of 4.5 and 11.5 weeks old $b /+$ and $b / b$ hearts with $2 \mathrm{G} 42 \mathrm{D} 7$ showed similar total myosin in the hearts.

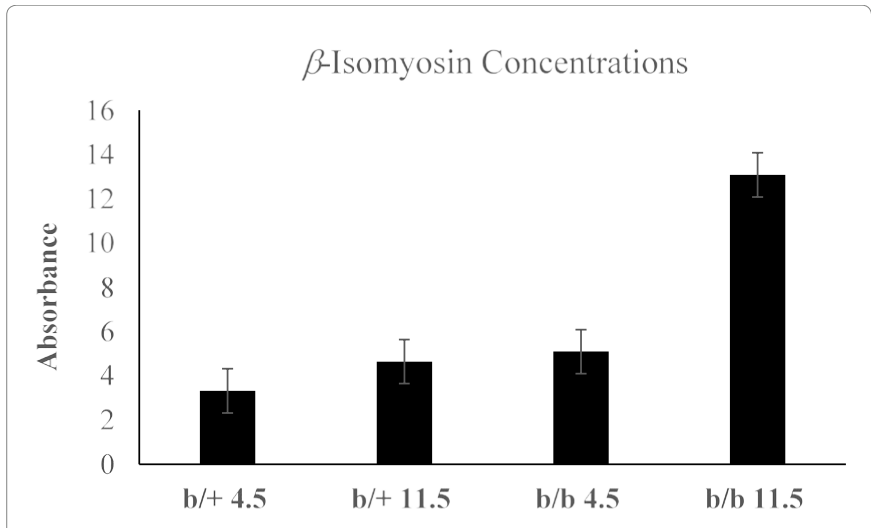

Figure 7. shows the absorbance of the immunohistochemical section of $b /+$ and $b / b$ rat hearts at 4.5 and 11.5 weeks of age stained with R11D10 monoclonal antibody specific for $\beta$-isomyosin. 


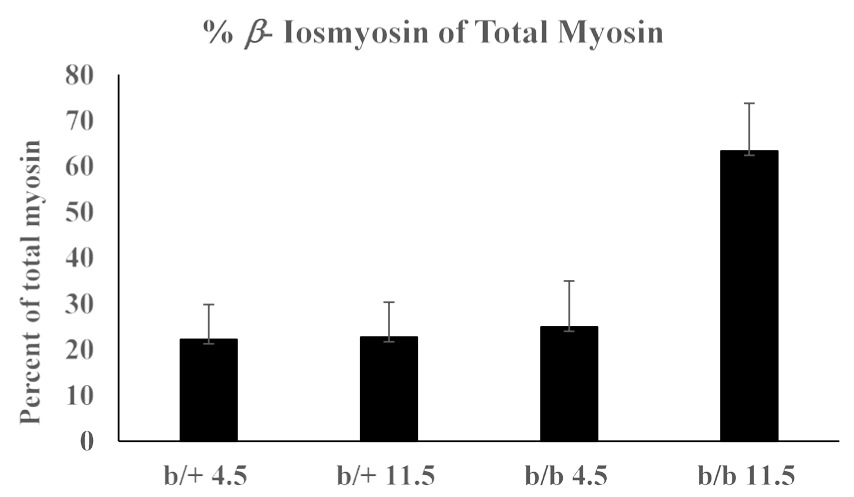

Figure $8 . \%$-isomyosin of total cardiac myosin in 4.5 and 11.5 weeks old $b /+$ and $b / b$ rats.

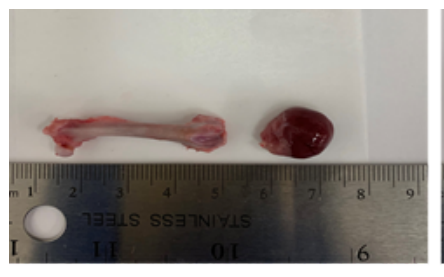

$b /+$
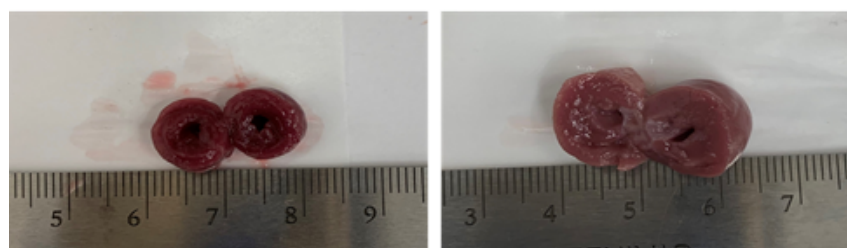

Figure 9. Heart tissues obtained from 11.5 weeks old $b /+$ rats and their age-matched $b / b$ rats. The femur is included as a measure of body size. The $b / b$ rats clearly demonstrate larger heart size and thicker cardiac muscle compared to $b /+$ rats.

than that of the $\beta$-isomyosin concentration in the 11.5 weeks old $b / b$ hearts.

\section{Discussion}

The $b / b$ rat is an animal model that shows a unique feature of iron loading anemia, which resembles thalassemia disorder in humans ${ }^{1,2,22}$. In thalassemia patients, cardiac complications are the primary cause of mortality and morbidity ${ }^{23,24}$. On the one hand, excess iron due to blood transfusion in thalassemia induces oxidative stress by generating noxious reactive oxygen species, which causes cardiomyocyte damage including apoptosis, fibrosis and cardiac dysfunction ${ }^{25}$ likely due to affecting the cardiac excitation-contraction coupling ${ }^{26}$. Although the production of hydroxyl radicals and lipid peroxidation is important in the initiation of iron-overload cardiomyopathy, subcellular organelles and inflammatory mediators are also believed to be involved in cardiac injury. For example, when iron overload occurs, proinflammatory mediators, such as
TNF- $\alpha$, MCP-1, and IL-6, express within the myocardium and induce cardiomyocyte apoptosis and cardiac fibrosis ${ }^{26}$. On the other hand, severe anemia leads to inadequate oxygen delivery to tissues. Therefore, the physiologic response to anemia promotes a compensatory increase in cardiac output in order to maintain adequate oxygen delivery, which could cause myocyte dysfunction. Our study indicates that $b / b$ rats display cardiomyopathy as assessed by the heart to body weight ratios compared to control heterozygous $b /+$ rats. Although non-heme iron did not change in $b / b$ rats, total heart iron in $b / b$ rats is significantly reduced compared to that of $b /+$ rats, suggesting that cardiac complications in $b / b$ rats were likely due to chronic anemia. Our results are consistent with Galanello et al., who reported that in patients with thalassemia intermedia, cardiac ventricles are enlarged without heart iron overload, possibly due to the hyperdynamic circulation associated with chronic anemia and to pulmonary hypertension ${ }^{27}$. Studies indicated that severe anemia could induce cardiac hypertrophy. For example, Paplanus et al. demonstrated that cardiac hypertrophy was found in anemic $\operatorname{dog} s^{28}$. In addition, the enlargement of heart in anemia could be corrected if the anemic condition is improved ${ }^{29}$. Our Belgrade $b / b$ rats displayed a significant increase of MHC- $\beta$ without changes in total MHC at 11.5-wk of age, as assessed by ELISA and immunohistochemical staining. Reiser et al. reported that during the progression of heart failure in humans, there is a change in the composition of MHC isoforms in ventricles and atria; the MHC- $\alpha$ expression is decreased, while the expression of MHC- $\beta$ is significantly increased ${ }^{30,31}$. Therefore, the changes in the MHC composition in Belgrade $b / b$ rats could serve as a biomarker for cardiac hypertrophy.

\section{Source of Funding}

This research was funded by the unrestricted funding of B.A. Khaw.

\section{References}

1. Sladic-Simic D, Martinovitch PN, Zivkovic N, et al. A THALASSEMIALIKE DISORDER IN BELGRADE LABORATORY RATS*. Ann N Y Acad Sci. 2006; 165: 93-99.

2. Thompson K, Molina RM, Brain JD, et al. Belgrade Rats Display Liver Iron Loading. J Nutr. 2006; 136: 3010-3014.

3. Bowen BJ, Morgan EH. Anemia of the Belgrade rat: evidence for defective membrane transport of iron. Blood. 1987; 70-44.

4. Gunshin H, Mackenzie B, Berger UV, et al. Cloning and characterization of a mammalian proton-coupled metal-ion transporter. Nature. 1997; 388: 482-488.

5. Fleming MD, Romano MA, Su MA, et al. Nramp2 is mutated in the anemic Belgrade (b) rat: Evidence of a role for Nramp2 in endosomal iron transport. Proc Natl Acad Sci USA. 1998; 95: 1148-1153.

6. Olivetti G, Lagrasta C, Quaini F, et al. Capillary growth in anemiainduced ventricular wall remodeling in the rat heart. Circ Res. 1989; 65: 1182-1192.

7. Khaw BA, Chan WT, Bhattarai $\mathrm{P}$, et al. Quantitative changes in $\beta$-isomyosin expression in the Belgrade rat model of hypertrophic 
cardiomyopathy: Assessment by ELISA and Immunoperoxidase quantitation. Submitted to SNMMI annual meeting. SNMMI Annual meeting 2016.

8. Mercadier JJ, Lompré AM, Wisnewsky C, et al. Myosin isoenzyme changes in several models of rat cardiac hypertrophy. Circ Res. 1981; 49: 525-532.

9. Sukumaran A, Chang J, Han M, et al. Iron overload exacerbates ageassociated cardiac hypertrophy in a mouse model of hemochromatosis. Sci Rep. 2017; 7.

10. Khaw BA, Mattis JA, Melincoff G, et al. Monoclonal antibody to cardiac myosin: imaging of experimental myocardial infarction. Hybridoma. 1984; 3: 11-23.

11. Sweadner KJ, McGrail KM, Khaw BA. Discoordinate regulation of isoforms of $\mathrm{Na}$,K-ATPase and myosin heavy chain in the hypothyroid postnatal rat heart and skeletal muscle. J Biol Chem. 1992; 267: 769773.

12. Torrance JD, Bothwell TH. A simple technique for measuring storage iron concentrations in formalinised liver samples. S Afr J Med Sci. 1968; 33: 9-11.

13. Brain JD, Heilig E, Donaghey TC, et al. Effects of Iron Status on Transpulmonary Transport and Tissue Distribution of Mn and Fe. Am J Respir Cell Mol Biol. 2006; 34: 330-337.

14. Kim J, Molina RM, Donaghey TC, et al. Influence of DMT1 and iron status on inflammatory responses in the lung. Am J Physiol Lung Cell Mol Physiol. 2011; 300: L659-L665.

15. Khaw BA, Beller GA, Haber E, et al. Localization of cardiac myosin-specific antibody in myocardial infarction. J Clin Invest. 1976; 58: 439-446.

16. Bradford MM. A rapid and sensitive method for the quantitation of microgram quantities of protein utilizing the principle of protein-dye binding. Anal Biochem. 1976; 72: 248-254.

17. Khaw BA, Beller GA, Haber E. Experimental myocardial infarct imaging following intravenous administration of Iodine-131 labeled antibody $\left(\mathrm{Fab}^{\prime}\right)_{2}$ fragments specific for cardiac myosin. Circulation. 1978; 57: 743-750.

18. Engvall E, Perlmann P. Enzyme-linked immunosorbent assay (ELISA) quantitative assay of immunoglobulin G. Immunochemistry. 1971; 8: 871-874.
19. Weemen BKV, Schuurs AHWM. Immunoassay using antigen-enzyme conjugates. FEBS Lett. 1971; 15: 232-236.

20. Sweadner KJ, McGrail KM, Khaw BA. Discoordinate Regulation of Isoform of Na, K-ATPase and Myosin Heavy Chain in the Hypothyroid Postnatal Rat Heart and Skeletal Muscle. J Biol Chem.1992; 267: 769773.

21. BioLegend. Immunohistochemistry Protocol for Frozen Sections.

22. Kim J, Jia X, Buckett PD, et al. Iron loading impairs lipoprotein lipase activity and promotes hypertriglyceridemia. FASEB J. 2013; 27: 1657 1663.

23. Wood JC, Enriquez C, Ghugre N, et al. Physiology and pathophysiology of iron cardiomyopathy in thalassemia. Ann N Y Acad Sci. 2005; 1054: 386-395.

24. Zurlo MG, De Stefano P, Borgna-Pignatti C, et al. Survival and causes of death in thalassaemia major. Lancet Lond Engl. 1989; 2: 27-30.

25. Gammella E, Recalcati S, Rybinska I, et al. Iron-induced damage in cardiomyopathy: oxidative-dependent and independent mechanisms. Oxid Med Cell Longev. 2015; 2015: 230182.

26. Cheng CF, Lian WS. Prooxidant Mechanisms in Iron Overload Cardiomyopathy. BioMed Res Int BioMed Res Int. 2013; 2013: 740573-740573.

27. Galanello R, Tanner M, Matta G, et al. Lack of Heart Iron Overload in Patients with Thalassemia Intermedia.. Blood. 2006; 108(11): 3822. Accessed May 01, 2018. Retrieved from http://www. bloodjournal.org/content/108/11/3822.

28. PAPLANUS SH, ZBAR MJ, HAYS JW. Cardiac Hypertrophy as a Manifestation of Chronic Anemia. Am J Pathol. 1958; 34: 149-159.

29. Ball, David. "Change in the size of the heart in severe anemia: With report of a case." American Heart Journal 6. 1931; 4: 517-521.

30. Reiser PJ, Moravec CS. Sex differences in myosin heavy chain isoforms of human failing and nonfailing atria. Am J Physiol Heart Circ Physiol. 2014; 307: H265-272.

31. Reiser PJ, Portman MA, Ning XH, et al. Human cardiac myosin heavy chain isoforms in fetal and failing adult atria and ventricles. Am J Physiol Heart Circ Physiol. 2001; 280: H1814-1820. 